\title{
A Conversation with Don Dawson
}

\section{Bouchra R. Nasri, Bruno N. Rémillard, Barbara Szyszkowicz and Jean Vaillancourt}

\begin{abstract}
Donald Andrew Dawson (Don Dawson) was born in 1937. He received a bachelor's degree in 1958 and a master's degree in 1959 from McGill University and a Ph.D. in 1963 from M.I.T. under the supervision of Henry P. McKean, Jr. Following an appointment at McGill University as professor for 7 years, he joined Carleton University in 1970 where he remained for the rest of his career. Among his many contributions to the theory of stochastic processes, his work leading to the creation of the Dawson-Watanabe superprocess and the analysis of its remarkable properties in describing the evolution in space and time of populations, stand out as milestones of modern probability theory. His numerous papers span the whole gamut of contemporary hot areas, notably the study of stochastic evolution equations, measure-valued processes, McKean-Vlasov limits, hierarchical structures, super-Brownian motion, as well as branching, catalytic and historical processes. He has over 200 refereed publications and 8 monographs, with an impressive number of citations, more than 7000. He is elected Fellow of the Royal Society and of the Royal Society of Canada, as well as Gold medalist of the Statistical Society of Canada and elected Fellow of the Institute of Mathematical Statistics. We realized this interview to celebrate the outstanding contribution of Don Dawson to 50 years of Stochastics at Carleton University.
\end{abstract}

Key words and phrases: Biography, probability, statistics, stochastic processes, physics, biology.

\section{INDIVIDUAL EXPERIENCE}

BN et al.: How did you initially get interested in the subject of probability?

Don: In my undergraduate student days, the role of entropy in thermodynamics captured my interest. The use of entropy in information theory and its applications to mathematical linguistics which was the subject of my M.Sc. thesis. Then at M.I.T., from 1959 to 1963, as a member of the Artificial Intelligence Project, I was in-

Bouchra R. Nasri is Assistant Professor, Département de médecine sociale et préventive, École de santé publique, Université de Montréal, C.P. 6128, succursale Centre-ville, Montréal (Québec) H3C 3J7, Canada (e-mail: bouchra.nasri@umontreal.ca).Bruno N. Rémillard is Honorary Professor, Department of Decision Sciences, HEC Montréal, 3000 Côte Sainte-Catherine, Montréal (Québec) H3T 2A7, Canada (e-mail: bruno.remillard@hec.ca). Barbara Szyszkowicz is Professor, School of Mathematics and Statistics, Carleton University, 1125 Colonel By Drive, Ottawa, Ontario, K1S 5B6, Canada (e-mail: bszyszko@math.carleton.ca). Jean Vaillancourt is Adjunct Professor, Department of Decision Sciences, HEC Montréal, 3000 Côte Sainte-Catherine, Montréal (Québec) H3T 2A7, Canada (e-mail:

jean.vaillancourt@hec.ca). spired by the course of Marvin Minsky in the new field of artificial intelligence which dealt with the uses of random methods for search and interacting systems. I took an inspiring course on the (now famous) book of Henry P. McKean Jr. and Kiyosi Itô: Diffusion processes and their sample paths (Itô and McKean, 1974) and decided to write my thesis on this topic.

BN et al.: Who were your mentors during your studies?

Don: At McGill University, Edward Rosenthall whose real analysis course was inspiring. Joachim "Jim" Lambek was the advisor for my M.Sc. thesis, and at M.I.T., my Ph.D. advisor was Henry P. McKean, Jr. I returned to McGill after completing my Ph.D. to take up a professorship. I taught at McGill University from 1963 to 1970 where I had four Ph.D. students. (I had two more at McGill later on.) Then in 1970 I came to Carleton University, where I supervised 23 Ph.D. students. I also worked with 35 postdoctoral fellows and research associates.

\section{EARLY YEARS}

BN et al.: You came from McGill to Carleton University in 1970 . What were your main motivations and the challenges you faced? 


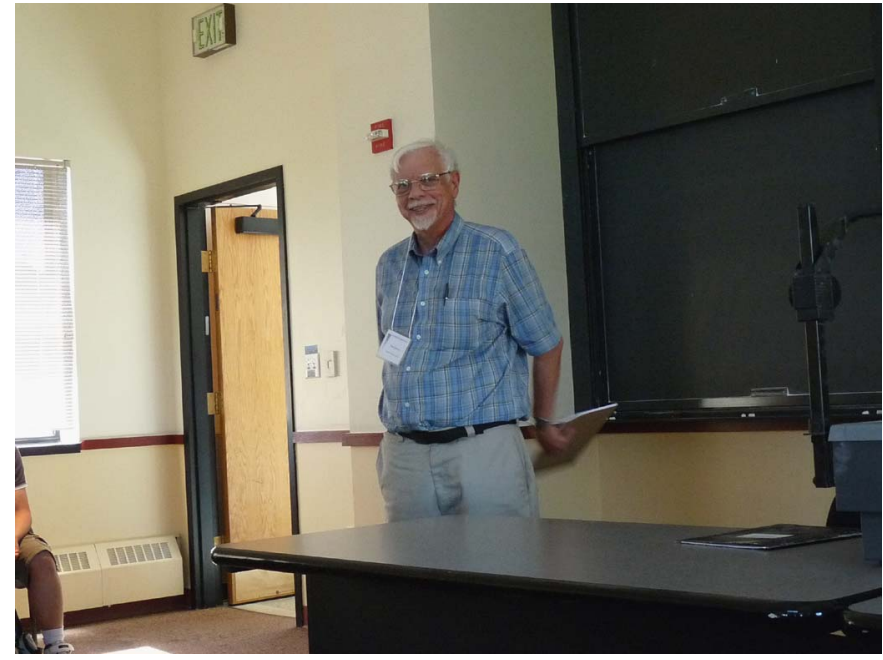

FIG. 1. Don Dawson during a conference in honour of Kuznestsov at University of Colorado (Boulder) in 2010.

Don: The main motivation was to build the Carleton Ph.D. program. The first challenge was having the assessment of the Ph.D. Program in Probability and Statistics. Fortunately, Miklós Csörgó came in 1971 and the two of us worked together, then a couple of years later Jon Rao came.

BN et al.: Talk to us about your early years at Carleton University.

Don: Many activities arose at Carleton during the 1970s. For example, there was the Symposium on Probability and Related Topics in 1973. Another was a series of lectures by Takeyuki Hida on the Analysis of Brownian Functionals in 1975, highlighted throughout by Hida's signature fans in his overhead talks. Also memorable were a series of lectures by Josef Steinebach on Large deviation probabilities and some related topics in 1980, and the International Symposium in Ottawa in 1980, for which the proceedings were published in 1981 by North-Holland.

BN et al.: How did the collaborations with the University of Ottawa emerge?

Don: At McGill, I had developed a close relationship with Anatole Joffe, who had studied with Mark Kac. Anatole's former student David McDonald and later my student Gail Ivanoff, as well as Mayer Alvo, who came from Columbia University, after a masters thesis at McGill, all became professors at the University of Ottawa. As a result, it was normal to have good relations between the two universities.

\section{LRSP YEARS}

BN et al.: The Carleton University-University of Ottawa Laboratory for Research in Statistics and Probability (LRSP) at Carleton University was a hive of research in probability and statistics from its creation in 1982 on.

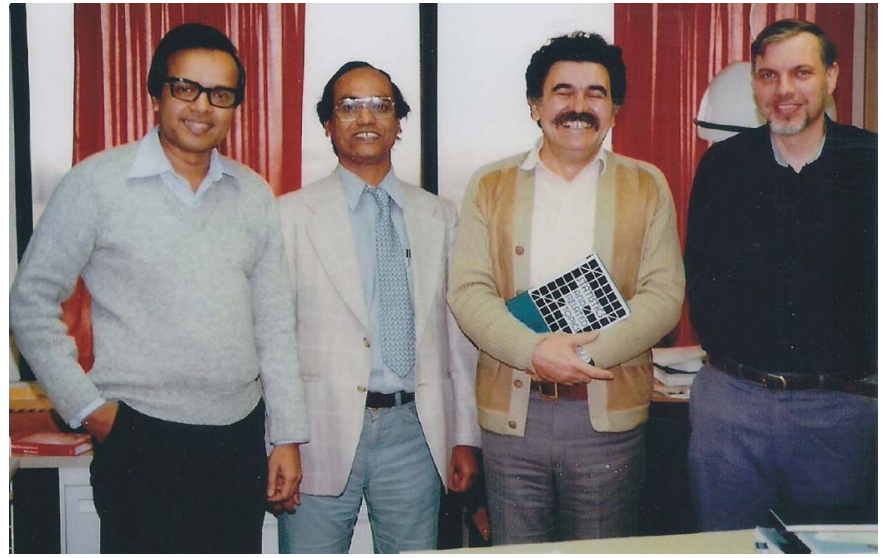

FIG. 2. Founders of the LRSP from Carleton University: from left to right, J.N.K. Rao, A.K.M.D.E Saleh, M. Csörgö and D. Dawson.

How did it come about and what were the main challenges to its creation?

Don: The main challenges were to find the appropriate funding. From 1983 to 1998, we obtained over 400K from the Natural Sciences and Engineering Research Council of Canada (NSERC). We also benefited from NSERC Conference grants and International Foreign Researcher grants.

BN et al.: Using rankings based on all research articles published between 1986 and 2000 inclusively in 25 select journals of high reputation (9 in Probability and 16 in Statistics), Genest and Guay (2002) identified the Carleton University Statistics and Probability group as one of only two in the world as having high productivity in both statistics and probability (the other one was Cornell), among the top 15 most productive statistical institutions in the world at the time. In a prior survey of the period 1986-1995 by Genest (Genest, 1999), the LRSP was one of three such groups (the other two were Cornell and the Université Libre de Bruxelles). What was the secret of the success of the LRSP? What are the key ingredients in your opinion?

Don: What made the LRSP so successful was the set of people who formed the team and the participation of a critical mass of researchers from Carleton University, the University of Ottawa and Statistics Canada. The key was a wide vision of the discipline of stochastics, and the scientific generosity of its members. The funding we received made possible the hiring of Mrs. Gill Murray as the Coordinator of the LRSP; she provided the outstanding coordination and was the glue, which made the wide range of activities possible.

BN et al.: Would it be possible to reproduce this success today? What are the barriers to realizing this again?

Don: Many things have changed since 1982. It is not clear to me what mechanism could produce this type of 


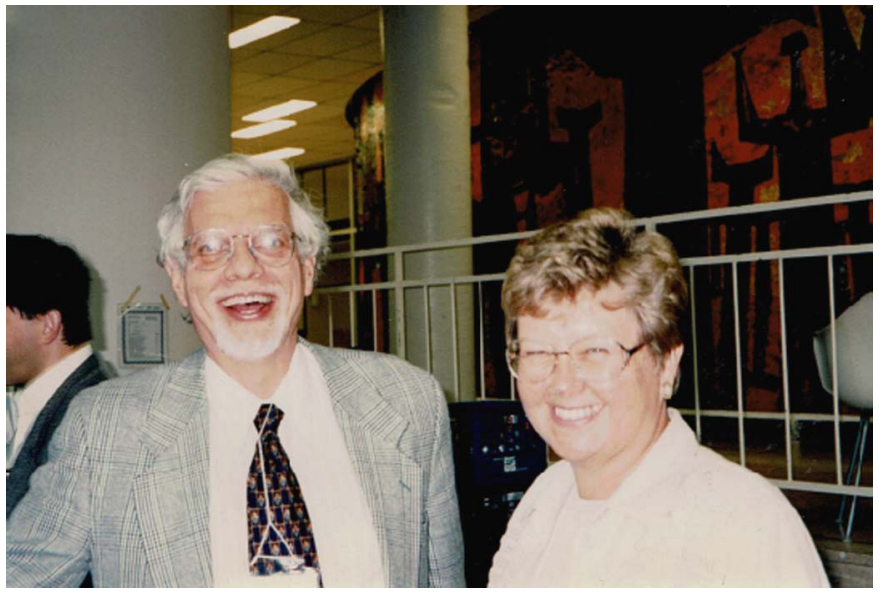

FIG. 3. Don Dawson and Gill Murray.

funding. However if this were possible, and the other conditions I mentioned above for this success of LRSP were met, it could be achieved.

BN et al.: Can you speak to us about the major contributions to our field that emerged around the laboratory, during that time and since? What was the impact of the numerous visitors on site? Can you name a few of the most distinguished visitors you received at the laboratory? Any defining moments (conferences or visits) or anecdotes (amusing or enlightening) to share with us?

Don: Our Technical Report Series was a real success with more than 450 volumes. Over the course of 50 or so years there are many enlightening and sometime amusing incidents involving visitors. I can highlight a few.

One of the most inspiring talks was given by Mark Kac, who was one of the first to explain mathematical physics and the Gibbs measure to probabilists like me. One of his favorite sayings was "a demonstration convinces a reasonable man whereas a proof satisfies a stubborn one."

Robert Adler who was used to the weather in Australia and Israel, faced a -30 degree (C) spell in Ottawa. He looked a little cold but it did not affect his impressive presentation. This included a lab demonstration of his software which later appeared as "Superprocesses-The Movie."

Jean-Francois Le Gall's blackboard talks, given as part of the Fields Institute thematic program on Probability and its Applications in August 1998-July 1999, were both inspiring and enlightening.

As time went by, mathematics talks went from blackboard to overhead slides and then to computer aided talks. A case in point were the superb lectures Tom Kurtz gave on the "look-down" process about his work with Peter Donnelly (Donnelly and Kurtz, 1996). This became one of the major advances in population genetics. His lecture was an excellent example of the good use of a computer talk. Another memorable lecture was given by
Terry Lyons on his seminal work introducing rough paths. Terry's was the first one I saw using a computer (tablet) as a "blackboard."

\section{COLLABORATIONS AND INTERACTIONS}

BN et al.: What are your favorite papers and why?

Don: I have listed my 3 favorite papers before 1991 because my future work, one way or another, was based on these three.

Dawson and Hochberg (1982) is a favorite of mine since it led to much of my research since then that has been focused on Fleming-Viot processes. Wendell Fleming was the External Examiner for Amit Bose's Ph.D. thesis defense in 1977 and he came to Carleton for this reason. We discussed his joint work with Viot and I became interested in their model. Ken Hochberg came to Carleton as a PDF (he was also supervised by H.P. McKean). With Ken, we saw that we could show that the moment measures satisfies de Finetti's theorem and to use this to reconstruct the measure. One application was to show that the long time behavior has a coherence property called which we called "wandering." I gave a talk at the Annual Winter Meeting of the Canadian Mathematical Society at Vancouver in December 1980 on a early draft and somebody pointed the connection that our wandering measure could be interpreted as a continuous limit of the of model of Kingman (1976). This also led to Dawson and Hochberg (1983).

Dawson and Gärtner (1987) is also a favorite of mine since it was a starting point for future applications of large deviation arguments in mean-field models. I first met Jürgen Gärtner in Berlin in 1986 while visiting Klaus Fleischmann. I was interested in McKean-Vlasov processes (Dawson, 1983) and Jürgen was an expert on large deviation theory. The purpose of this paper was to show that the numbers of particles $N \rightarrow \infty$ which is given by the McKean-Vlasov equation and the large deviation behavior is described by a generalization of the Freidlin and Wentzell results to obtain a characterization of the action functional. This is achieved by a projective limit of large deviation systems, large deviations on dual vector spaces and a Sanov type theorem for vectors of empirical measures. I coauthored several papers with Jürgen including a AMS memoir and lectures at the Cambridge Symposium in 1987, "Stochastic calculus in application." I learned a great deal from him. Based on Google searchers, there were a surprising numbers of citations on mean-field results and their large deviations limits as the number of particles goes to $\infty$. This includes stochastic dynamic games, stochastic filtering, stochastic control, stochastic spin systems, ecology, nonequilibrium thermodynamics and random polymers.

Another favorite of mine is Dawson and Perkins (1991) since it brought together the work done on superprocesses 
during the period 1968 and 1990 by Watanabe, Iscoe, Perkins and me by introducing the historical processes as a superprocess, which is enriched to contain information on the genealogy of the population.

BN et al.: How did historical processes come about? You cowrote major papers on the subject with Ed Perkins. Can you speak in more detail about this collaboration?

Don: The subject began with Watanabe (1968). Unfortunately, I was unaware of this article until I read Walsh's Saint-Flour notes (Walsh, 1986), which refers to it. My work on superprocesses was originally motivated by the dimensional dependence of stochastic evolution equations in Euclidean spaces (Dawson, 1972, 1975). In my 1975 paper, the stochastic evolution equation on branching results in a measure-valued process in any dimension. This is based of the use of the Trotter product formula. Dawson (1977, 1978/79, 1979), Dawson and Hochberg (1982), those of Iscoe (1981, 1986a, 1986b, 1988) (who had developed a formula for the occupation time of a superprocess in his Carleton Ph.D. thesis) and Perkins $(1988,1989,1990)$ also explore many questions of the dimension dependence.

The notion of the historical process came about in early discussions we had with Ian Iscoe. The formulations of historical processes that took place in the sixth floor of the Dunton Tower at Carleton University slowly came together using the general superprocess due to Fitzsimmons, the use the nonstandard characterization of weak convergence, which was new to me and the Palm measure formula and Campbell measure. The latter was based on the theory of infinitely divisible random measures of Jagers (1974), Kallenberg (1978), Matthes, Kerstan and Mecke (1978).

Over the next 15 years, I had the pleasure to work on several projects with Ed. One example of this is our joint work on mutually catalytic branching. During that time, we were stuck on finding the dual-fortunately Leonid Mytnik produced new dual. In turn, this led to the five author collaboration (Dawson et al., 2002). One memorable meeting of the authors was at the Fields Institute. Over the years, Perkins has made the many of the deepest results in superprocess. His Saint-Flour Notes (Perkins, 2002) contain the state of the art before 2003. I have been also influenced in particular by his later joint work with Steve Evans (Evans and Perkins, 1998). This, together with Barlow and Perkins (1994), played an important role in Dawson and Fleischmann (1997).

BN et al.: Can you talk to us about your relationship over the years with Dynkin and Le Gall? The publications by Dynkin and Le Gall in the field came much later and the main ones are both dated in 1991. What can you say about their contribution to our understanding of superprocesses and how superprocesses came to bear your name, as in Dawson-Watanabe processes?

Don: In the late 1980s, Dynkin became interested in superprocesses. I first leaned of this by an email message asking questions about my 1978 paper. Later he also arranged a visit by me and also Iscoe to Cornell. I was invited to give one of the Barrett lectures at the University of Tennessee in 1993. Dynkin was one of the other lectures there where I learned of his new results; these also formed the contents of Dynkin (1994). Over the years, Dynkin made many contributions to superdiffusions including the important notion of branching exit Markov systems. This and many more deep results of his are presented in Dynkin (2002). Dynkin's branching rate functional played an essential role in Dawson and Fleischmann (1997). Dynkin introduced the name superprocesses in Dynkin (1988) and the name Dawson-Watanabe processes in the book Dynkin (1994). He later introduced the class of superprocesses $(\xi, K, \psi)$. The superprocess with $\xi$ a Brownian motion, quadratic branching and $K(t) \propto d t$ was called the Dawson-Watanabe superprocess.

Le Gall presented his Brownian excursion, trees and measure-valued branching processes paper (Le Gall, 1991) at the Workshop on Infinite dimensional Markov processes at Cornell University in May 1989. This paper develops a representation based on a tree inspired by Neveu and its relation to the historical process. It is a powerful tool for the superprocess and their generalizations. His basic construction is extended in Duquesne and Le Gall (2002) to Lévy processes to produce the Lévy snake. One application is to get the stable analogue of Aldous' continuum Brownian random tree (Aldous, 1991a, 1991b, 1993). The Brownian snake also was used in the Habilitation Dissertation of J.-F. Delmas, for which I was asked to report in 1997. It included a section on catalytic branching including his paper with Fleischmann. His work has influenced me in several ways. In 2002, we used the Brownian snake to discuss strong clumping in a stable catalytic medium (Dawson, Fleischmann and Mörters, 2002). Also the stochastic flow results of Bertoin and Le Gall (2003), Bertoin and Le Gall (2005) were the inspiration for Dawson and Li (2012).

BN et al.: Other than Dynkin, Le Gall and Perkins, which three other researchers would you consider to have written the most influential papers in your field or fields of endeavor? How did they influence your own research?

Don: I will mention Allison Etheridge, Tom Kurtz and Tokuzo Shiga. Barton, Etheridge and Véber (2010) was the beginning of an approach to evolution in a spatial continuum that has since had an important impact on the subject. Etheridge (2011) and subsequent work since is a major contribution to evolutionary biology. I have followed 


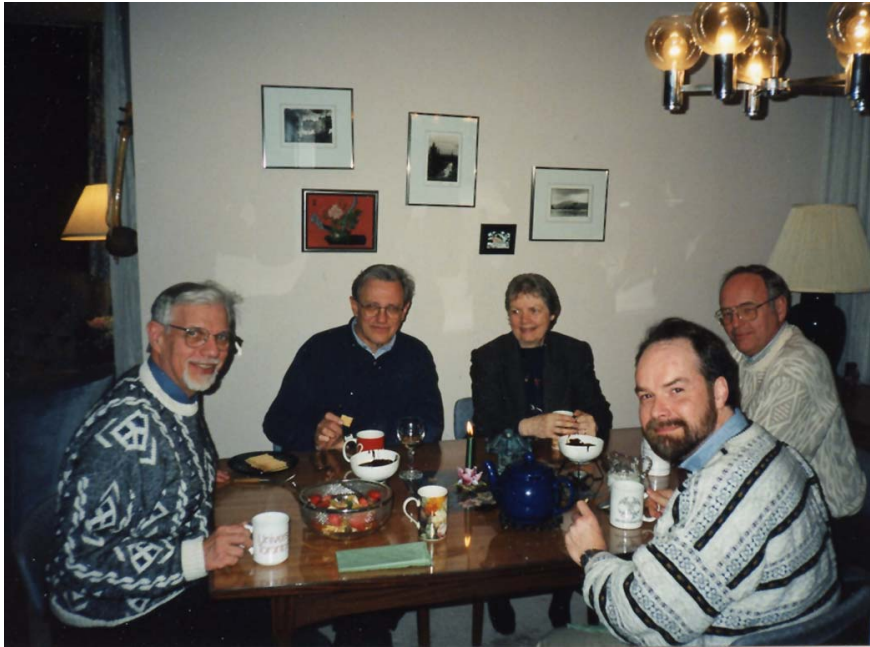

FIG. 4. From left to right: Don Dawson, Luis Gorostiza, Caroline Kurtz, Tom Kurtz and Ed Perkins.

the work of Etheridge since her Ph.D. thesis. Etheridge and March (1991) was the first to point out the relationship between superprocesses and the Fleming-Viot process, which was an important step. We were coauthors on Dawson et al. (2002).

Donnelly and Kurtz (1996) was the start of a development which represents the genealogy of a Fleming-Viot populations and it had a major impact on population biology. I spent my sabbatical during 1980-1981 visiting the University of Wisconsin. Kurtz and his former student Ethier were starting to work on Ethier and Kurtz (1986), which has had a major impact in the field of Markov processes. At that time, I was completing the joint paper with Ken Hochberg on the Fleming-Viot process, which had introduced the dual method for measure-valued processes. In a train ride from Madison to Chicago, we started to work on our joint paper on the general conditions necessary for the duality. Greven and I used the results of Ethier-Kurtz and especially the results on coupling and ergodic theorems.

I spent the period October-November 1994 visiting Shiga at the Tokyo Institute of Technology and learned much from him. I was familiar with his earlier paper Shiga and Shimizu (1980) on infinite-dimensional stochastic differential equations, when he gave a series of lectures at Carleton, up to Konno and Shiga (1988). But I was unaware of his earlier work (Shiga and Uchiyama, 1986) which I learned during this visit. This paper provided some of the key tools in my later work with Greven on Fleming-Viot processes (Dawson and Greven, 1999, 2014).

BN et al.: Can you talk to us about your relationship over the years with your long term collaborators Klaus Fleischmann, Luis Gorostiza and Andreas Greven?

Don: I first met Klaus Fleischmann at a meeting in Hungary in 1978. We had a long collaboration. We

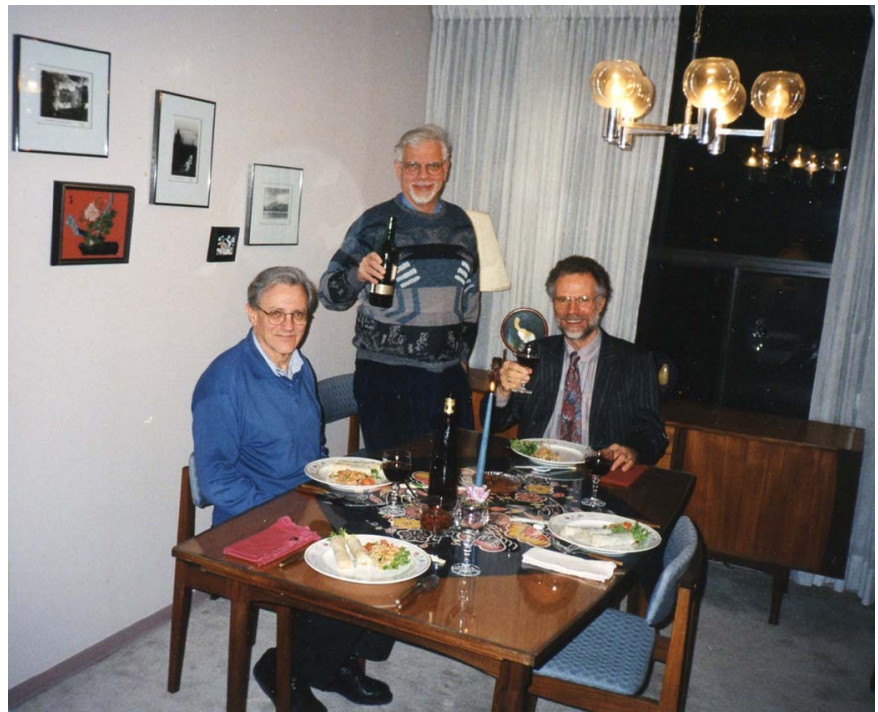

FIG. 5. From left to right: Luis Gorostiza, Don Dawson and Klaus Fleischmann.

worked on catalytic and mutually catalytic superBrownian motions, including the work mentioned previously. Among my favorites is Dawson and Fleischmann (1997).

Luis Gorostiza is another one of my long term collaborator. Our first joint paper was in 1984 (Dawson and Gorostiza, 1984). We worked on a variety of topics, including Dawson, Gorostiza and Li (2002), and Dawson, Gorostiza and Wakolbinger (2001) where, among other things, we identify the order of transience and recurrence of hierarchical processes. Most recently, we worked on random walks and percolation on Euclidean and hierarchical systems and their continuum limits (Dawson and Gorostiza, 2018). Highlights were our trips to Beijing and CIMAT in Guanajuato.

If the metric is number of pages published together, then Andreas Greven and I are definitely the closest of collaborators. My collaboration in the early years with Andreas started with a Colloquium he gave at Carleton on his joint work with Ted Cox (Cox and Greven, 1994), and discussions we had afterwords. In 1995 at Carleton, we collaborated with Jean Vaillancourt on equilibria and quasiequilibria for infinite systems of interacting systems Fleming-Viot processes (Dawson, Greven and Vaillancourt, 1995). From May to July 2001, I was the Otto Haupt Visiting Professor at the University of ErlangenNürnberg and I had the pleasure to give a class to Greven's graduate students. In the last 20 years, Greven and his students Achim Klenke, Peter Pfaffehuber and Anita Winter had produced deep results on superprocesses and their generalizations. In 2008 at EURANDOM, we studied the renormalization transformation for two type branching models (Dawson et al., 2008). Our work on FlemingViot models with selection and mutation culminated with Dawson and Greven (2014). This constructed a rigorous 


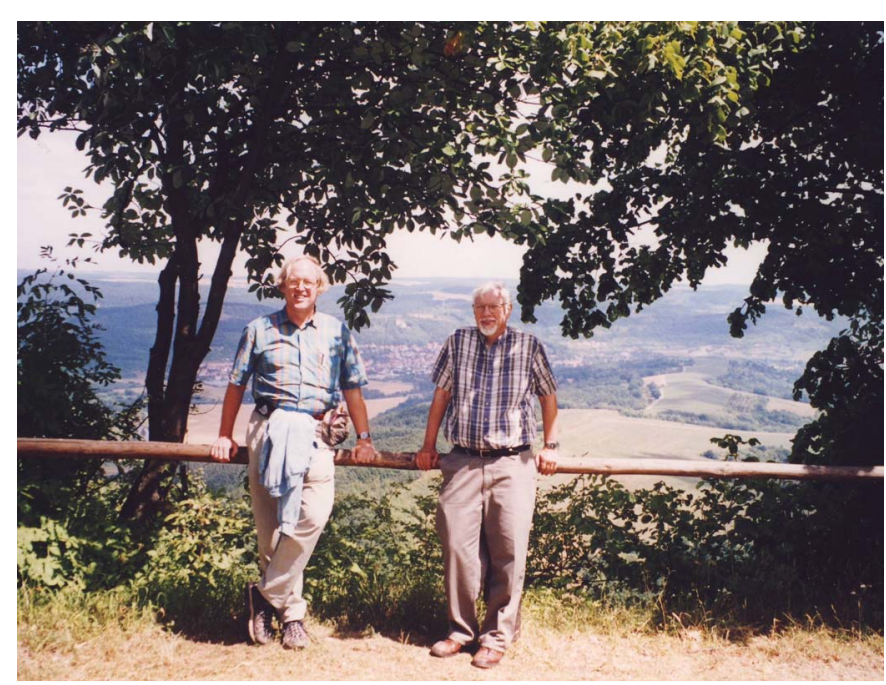

FIG. 6. Andreas Greven and Don Dawson.

framework for the analysis of the evolution of population toward fitter types though punctuated equilibria. We also taught a joint minicourse in 2017 based on this book at EURANDOM.

\section{SCIENTIFIC CONTRIBUTIONS}

BN et al.: You have published more than 200 papers. Many of your contributions aim at describing and analyzing the behavior of branching particle systems. Can you describe for our readers how your interest for these arose?

Don: This arose out my interest in the theory of the stochastic heat equation

$$
\frac{\partial X}{\partial t}=\frac{\partial^{2} X}{\partial x^{2}}+\alpha X^{\beta} \frac{\partial B}{\partial t}
$$

with $\beta \in(0,1]$ and $B(t)$ is standard Brownian motion. The case $\beta=\frac{1}{2}$ represents continuous state branching. The starting point was Dawson (1972) in which existence and uniqueness for Hilbert-space valued solutions for a class of parabolic stochastic partial differential equations (SPDE) with Lipschitz coefficients and the joint continuity in space and time for the stochastic heat equation in one dimension were established. In Dawson and Salehi (1980), it was noted that the linear SPDE $d X(t, x)=\Delta X(t, x)+X(t, x) d W(t, x)$ with $X(0, x) \geq$ 0 , now known as the parabolic Anderson equation, with space-time white noise $\mathrm{W}$ does not have a function-valued solution in $R^{d}$ for $d \geq 2$. However, it was proved using Itô-Wiener chaos expansions that function-valued solutions in $d \geq 2$ exist if the white noise is replaced by noise white in time but suitably colored in space.

The motivation for the development of measure-valued processes came from the observation that the Feller diffusion (continuous state branching) in dimensions $d \geq$ $2, d X(t, x)=\Delta X(t, x)+(X(t, x))^{1 / 2} W(d t, d x)$, where $W(\cdot, \cdot)$ denotes space-time white noise, does not have a function-valued solution but does have a measure-valued solution. This problem was addressed in Dawson (1975) by reformulating it as a measure-valued process for which existence and uniqueness in law was established. The resulting process now known as super-Brownian motion or Dawson-Watanabe superprocess had also been discovered earlier by S. Watanabe in the context of continuous state branching processes.

BN et al.: Your work on branching particle systems led to the introduction of families of measure-valued processes, which grew into a whole new field within probability theory. What are the results you are most proud of in this area?

Don: During the years 1970-1995, my research program focused on the characterization and classification of basic classes of measure-valued processes and the study of the small spatial scale and large space-time scale behavior of these processes. This led to the systematic development of the foundations of measure-valued processes presented in Dawson $(1993,2017)$, which now have important applications in genetics, ecology and other fields. For other measure-valued processes whose states are not infinitely divisible random measures, dual representations, that had applications to infinite particle systems, also could be applied to some measure valued processes. In Dawson and Hochberg (1982), we developed the method of duality for the probability measurevalued processes introduced by Fleming and Viot, as well as a particle representation and decomposition into a tree of subfamilies. Duality was also a key ingredient in a joint paper with George Papanicolaou (Dawson and Papanicolaou, 1984) for a Hilbert space-valued random Schrödinger process. The duality method was also reformulated in an abstract setting in a joint paper with Tom Kurtz (Dawson and Kurtz, 1982) and this has now become a standard tool.

Another basic tool is the historical superprocess and its canonical representation, which we introduced in a joint paper with E.A. Perkins (Dawson and Perkins, 1991) and used there to establish a number of structural properties of super-Brownian motion including the clan decomposition of the equilibrium distribution.

A method that can be used to handle a class of nonlinear interactions is the Dawson-Girsanov formula for measure-valued processes that we derived in Dawson (1978) and has been used to incorporate selection in Fleming-Viot processes and nonlinear birth and death rates in superprocesses. Some results for nonlinear sampling rates for Fleming-Viot sufficiently close to constant rates were obtained in a joint paper with P. March (Dawson and March, 1995) using a class of resolvent estimates and a semigroup perturbation argument.

BN et al.: What were the main applications driving the research on measure-valued processes? 
Don: The are so many applications. Here are a few.

Catalytic and mutually catalytic processes: A catalytic particle system is one in which the reactant population produces new particles only in the presence of a catalyst. A class of catalytic superprocesses was introduced and studied in a series of joint papers with Klaus Fleischmann. An example of that is Dawson and Fleischmann (1997) where a super-Brownian motion is catalyzed by another super-Brownian motion, which is nondegenerate only in dimensions $d=1,2,3$. Similar analysis can be carried out for catalytic chains having no cycles but chains having cycles are fundamentally different. The case of a 2-cycle (mutually catalytic branching) in which each of two types catalyzes the production of the other was introduced in Dawson and Perkins (1998). In the case of one spatial dimension existence and weak uniqueness was obtained with E.A. Perkins (Dawson and Perkins, 1998) for the associated pair of SPDE. Existence and uniqueness in law for the corresponding martingale problem in $R^{2}$ was established in a series of three papers in collaboration with A. Etheridge, K. Fleischmann, L. Mytnik, E.A. Perkins and J. Xiong but remains an open problem for $d \geq 3$.

Analysis of interacting systems in large space and time scales: A central problem in both statistical physics and population biology is the determination of the longtime behavior and possible equilibrium states for interacting systems on the lattice $Z^{d}$ (or other spatial network topology) with homogeneous initial conditions. We developed a program to determine such behavior for interacting diffusions, superprocesses, catalytic systems (Cox, Dawson and Greven, 2004) and interacting Fleming-Viot processes (Dawson and Hochberg, 1982) and developed methods for this purpose including McKean-Vlasov limit dynamics, large deviations for measure-valued processes and the hierarchical mean-field limit.

Large scale structures in measure-valued processes: Dawson (1977) resolved the problem of the existence of spatially homogeneous equilibria for critical branching systems in $\mathbb{R}^{d}$ by identifying a dichotomy in which local extinction and the formation of rare clumps occurs in dimensions $d=1,2$ and nontrivial equilibria exist in dimensions $d \geq 3$. Dawson and Perkins (1998) establish the same dichotomy for the mutually catalytic system on the lattice $\mathbb{Z}^{d}$, Dawson and Greven (1999) establish this for the interacting Fleming-Viot system and Dawson, Gorostiza and Wakolbinger (2004) for multilevel branching (but with critical dimension $d=4)$.

Mean-field methods and large deviations: Finally, mean-field models are used to capture major features of the behavior such as the phase transition structure. They arise in the McKean-Vlasov limit of a measure-valued system in which the spatial interaction is replaced by $N$ exchangeably interacting systems and then analyzing the $N \rightarrow \infty$ limit. An application of mean-field methods to queueing systems in given in Dawson, Tang and Zhao (2005). In Dawson (1983), we used the limiting McKeanVlasov dynamics to study phase transitions, critical slowing down and the emergence of non-Gaussian fluctuations at the critical point. The study of the large deviations for interacting systems and measure-valued processes was initiated in a series of joint paper with Jürgen Gärtner. This was used in Dawson and Gärtner (1987) to derive Wentzell-Freidlin estimates for deviations from the McKean-Vlasov limit in order to investigate the transition between quasi-equilibria in large finite systems. Large deviation methods are used in Dawson-Feng, including Dawson and Feng (2006), to study asymptotics for the Fleming-Viot process in the infinite population limit.

BN et al.: What do you see as future applications or problems for measure-valued processes?

Don: Several show intense research activity as we speak: mean field games and spatial mean field games, spatial epidemic models, ecology and evolutionary biology, multilevel systems, hierarchical network theory, random wave propagation, affine processes in finance.

BN et al.: Much of your later research production is concerned with applications to evolutionary biology. Tell us about hierarchical particle systems you and your collaborators introduced in order to model these extremely complex phenomena.

\section{Don:}

Hierarchical analysis and the degree of random walks: Hierarchical systems play a role in many fields such as evolutionary biology, neuroscience, protein dynamics, statistical physics and Bayesian statistics. Our research on this began jointly with Andreas Greven (Dawson and Greven, 1993). In turn, this led to our interest in the potential theory of random walk on groups in which we introduced the notion of degree of random walks. The results on branching on the hierarchical group based on the degree of random walks joint research are presented with Luis Gorostiza and Anton Wakolbinger (Dawson, Gorostiza and Wakolbinger, 2001).

Hierarchical mean-field analysis on the hierarchical group was introduced in joint work with Andreas Greven (Dawson and Greven, Dawson and Greven, 1993, 1999), and used to study interacting systems including branching systems, interacting Fleming-Viot processes (Dawson and Greven, 1999) and catalytic systems (Cox, Dawson and Greven, 2004) in multiple space and time scales and their dependence of the degree of the underlying random walk.

This research program has the objective of proving that the large scale behavior of spatially interacting sys- 
tems can be classified into a small number of universality classes (Dawson et al., 2008).

Application to evolutionary biology and ecology: Evolutionary biology is based on a complex adaptive system in which reproduction of individuals, subject to mutation and selection located on a space of sites, combine with migration between sites, given by a random walk in a spatial environment. This can be modeled by spatial Fleming-Viot systems with mutation and selection on a fitness landscape with its ultrametric structure. We consider, in particular, the emergence of new species. The analysis of this system and its behaviour in the long time scales involved, made it necessary to find a new family of dual processes, the set-valued ones. This was carried out in the book with Andreas Greven (Dawson and Greven, 2014).

Much current interest has developed in recent years in the question of levels of selection in evolutionary biology and evolutionary ecology. The corresponding natural framework for multilevel multitype models with random effects at different levels is the setting of multilevel measure-valued processes. In particular, two-level measure-valued processes have state spaces of the form $\mathcal{M}(\mathcal{M}(E))$ for some Polish space $E$ where $\mathcal{M}(E)$ denotes the space of Borel measures on $E$. In Dawson (2018), we work with an analogous class of two level probability measure-valued processes formulated in terms of a well-posed martingale problem which generalizes the two-type model to systems with more than two types, more complex interactions and to the diffusion limit of systems with finitely many sites. The application of this framework to evolutionary biology and ecology of multispecies systems in which the fitness of a species can depends on the higher level distributions of related species is given in Dawson (2018).

The problem of extending this analysis to spatially random environments modelled by migration between sites given by a nearest neighbor random walk on a network of long-range percolation clusters. This was recently carried out in ultrametric spaces in joint research with Luis Gorostiza (Dawson and Gorostiza, 2018).

\section{ACADEMIC DUTIES}

BN et al.: How did you reconcile this prolific productivity with your heavy involvement in editorial duties, directorships, grant agencies committee work, etc.?

Don: My main responsibilities were Department Chair (1974-1977), Coeditors-In-Chief of the Canadian Journal of Mathematics (1988-1993), 5 terms of NRC and NSERC committees during 1974-1995, Director of the Fields Institute (1996-2000) and President of the Bernoulli Society (2003-2005). During many of these years,

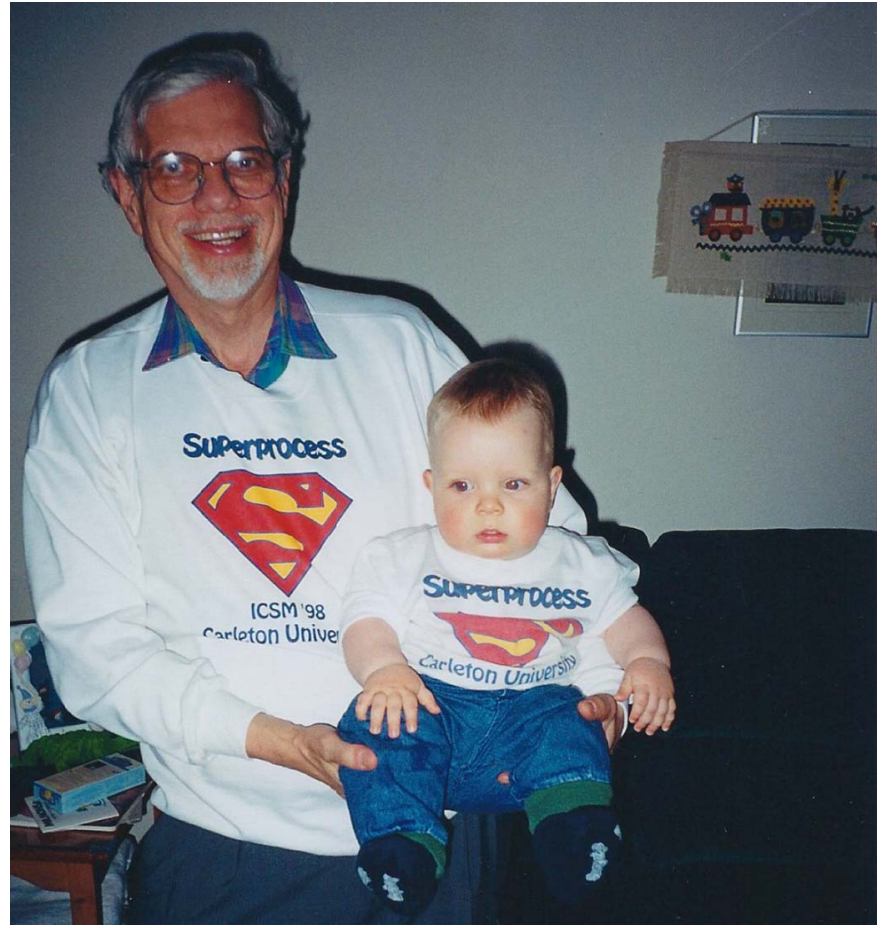

FIG. 7. Don with his grandson Andrew in July 1998.

my graduate students and coauthors kept me alive scientifically.

BN et al.: During your presidency of the Bernoulli Society, can you identify one or two key events of note to the community that you had a hand in?

Don: Three events do stand out. First is the 6th Bernoulli-IMS World Congress in Probability and Statistics, held in Barcelona in 2004. There were great plenary lectures by Jun Liu, Steffen L. Lauritzen, David Aldous, Wendelin Werner, Iain Johnstone, Peter Bickel, Vladimir Koltchinskii, Evarist Giné, Cun-Hui Zhang, Alison Etheridge and Dominique Picard. Quite a roster! This was followed by an excellent European Meeting of Statisticians in Oslo (2005). Last but not least, the agreement with the IMS called "Joined-up Thinking," the creation of a joint Bernoulli Society/IMS membership beginning in 2005. It seems natural now, given that the two societies share common objectives, namely the advancement of probability and statistics and their applications.

BN et al.: What is the role of your colleagues, friends and family in your professional successes?

Don: My wife Elizabeth (Betty) and children Michael and Suzanne have made everything possible. My colleagues, my students, my coauthors and visitors provided the environment, which were instrumental to my research program. I owe my coauthors a special thanks.

\section{PROSPECTIVE QUESTIONS}

BN et al.: Where do you see the field heading? 
Don: Randomness is ubiquitous and has produced many diverse many manifestations. Examples arise in the analysis of probabilistic models for real-world phenomena from physics, biology, neuroscience, statistics and computer science. Some specific topics of current interest are: statistical physics in a random environment, random matrix theory, branching systems in biology, random energy landscapes, data structure analysis, genetics and population biology, stochastic partial differential equations, networks and random walks on random graphs.

BN et al.: What do you see as the future of probability in the world of Artificial Intelligence and Big Data?

Don: These fields are multidisciplinary and require team work. This includes probability which is involved in hierarchical Bayes networks, random search, random landscapes, statistical physics and deep learning algorithms in data sciences.

BN et al.: What is your assessment of the current state of health of the field of stochastics in Canada and around the world?

Don: Stochastics has been developed in many directions over the past 50 years and is still going strong. Canada plays an important role in several areas and LRSP itself has contributed much to this success. Stochastics continues to generate important gains in health science, social science, economics, physics, genetics, evolutionary biology, neuroscience, interacting systems and networks, analysis of algorithms, financial probability and our general scientific knowledge on the whole.

BN et al.: Do you have any advice for the young researcher in probability.

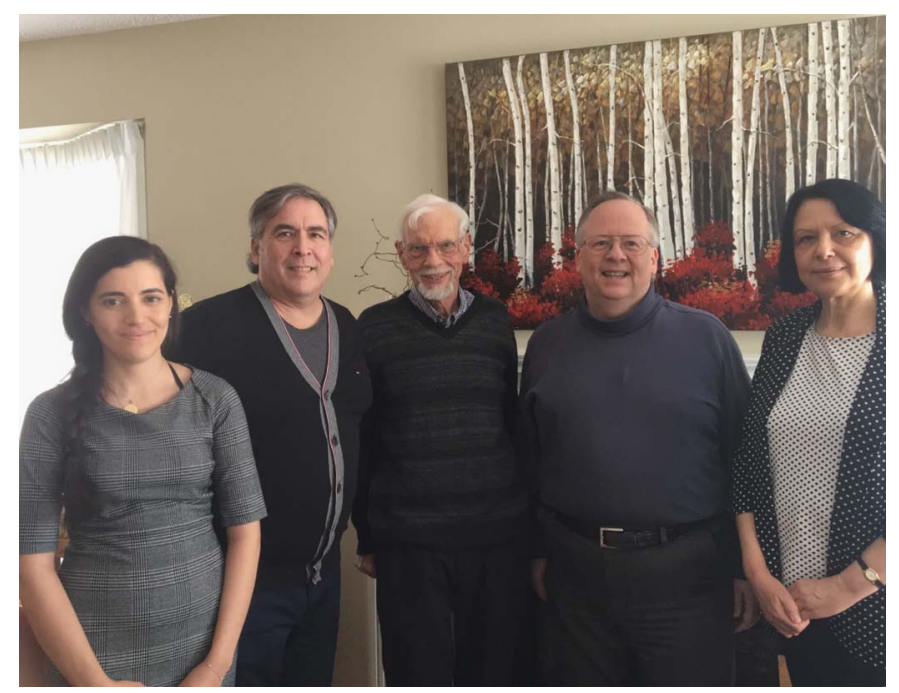

FIG. 8. Don Dawson and authors, February 28, 2020: from left to right, Bouchra R. Nasri, Bruno N. Rémillard, Don Dawson, Jean Vaillancourt and Barbara Szyszkowicz.
Don: Develop your set of mathematical skills. This can provide a foundation in future years as you broaden your interests. Keep your eyes open for new opportunities, for new applications and for scientific cooperation.

The interested reader can find out more by going to http://dynkincollection.library.cornell.edu/node/954.

\section{REFERENCES}

Aldous, D. (1991a). The continuum random tree. I. Ann. Probab. 19 1-28. MR1085326

Aldous, D. (1991b). The continuum random tree. II. An overview. In Stochastic Analysis (Durham, 1990). London Mathematical Society Lecture Note Series 167 23-70. Cambridge Univ. Press, Cambridge. MR1166406 https://doi.org/10.1017/CBO9780511662980. 003

Aldous, D. (1993). The continuum random tree. III. Ann. Probab. 21 248-289. MR1207226

Barlow, M. T. and Perkins, E. A. (1994). On the filtration of historical Brownian motion. Ann. Probab. 22 1273-1294. MR1303645

Barton, N. H., Etheridge, A. M. and Véber, A. (2010). A new model for evolution in a spatial continuum. Electron. J. Probab. 15 162-216. MR2594876 https://doi.org/10.1214/EJP.v15-741

Bertoin, J. and Le GALL, J.-F. (2003). Stochastic flows associated to coalescent processes. Probab. Theory Related Fields 126 261288. MR1990057 https://doi.org/10.1007/s00440-003-0264-4

Bertoin, J. and Le GALl, J.-F. (2005). Stochastic flows associated to coalescent processes. II. Stochastic differential equations. Ann. Inst. Henri Poincaré Probab. Stat. 41 307-333. MR2139022 https://doi.org/10.1016/j.anihpb.2004.07.003

Cox, J. T., Dawson, D. A. and Greven, A. (2004). Mutually catalytic super branching random walks: Large finite systems and renormalization analysis. Mem. Amer. Math. Soc. 171 viii+97. MR2074427 https://doi.org/10.1090/memo/0809

Cox, J. T. and Greven, A. (1994). The finite systems scheme: An abstract theorem and a new example. In Measure-Valued Processes, Stochastic Partial Differential Equations, and Interacting Systems (Montreal, PQ, 1992). CRM Proc. Lecture Notes 5 55-67. Amer. Math. Soc., Providence, RI. MR1278282 https://doi.org/10.1214/ aop/1176988732

DAWson, D. A. (1972). Stochastic evolution equations. Math. Biosci. 15 287-316. MR0321178 https://doi.org/10.1016/0025-5564(72) 90039-9

Dawson, D. A. (1975). Stochastic evolution equations and related measure processes. J. Multivariate Anal. 5 1-52. MR0388539 https://doi.org/10.1016/0047-259X(75)90054-8

DAwson, D. A. (1977). The critical measure diffusion process. Z. Wahrsch. Verw. Gebiete 40 125-145. MR0478374 https://doi.org/10.1007/BF00532877

Dawson, D. A. (1978). Geostochastic calculus. Canad. J. Statist. 6 143-168. MR0532855 https://doi.org/10.2307/3315044

DAwson, D. A. (1978/79). Critical behavior of the geostochastic logistic system. C. R. Math. Rep. Acad. Sci. Can. $179-82$. MR0519528

Dawson, D. A. (1979). Stochastic measure diffusion processes. Canad. Math. Bull. 22 129-138. MR0537294 https://doi.org/10. 4153/CMB-1979-020-3

DAWson, D. A. (1983). Critical dynamics and fluctuations for a mean-field model of cooperative behavior. J. Stat. Phys. 31 29-85. MR0711469 https://doi.org/10.1007/BF01010922 
DAwson, D. A. (1993). Measure-valued Markov processes. In École D'Été de Probabilités de Saint-Flour XXI-1991. Lecture Notes in Math. 1541 1-260. Springer, Berlin. MR1242575 https://doi.org/10.1007/BFb0084190

DAWSON, D. A. (2017). Introductory lectures on stochastic population systems. Preprint. Available at arXiv:1705.03781.

DAwson, D. A. (2018). Multilevel mutation-selection systems and set-valued duals. J. Math. Biol. 76 295-378. MR3742789 https://doi.org/10.1007/s00285-017-1145-2

DAwSON, D. A. and FENG, S. (2006). Asymptotic behavior of the Poisson-Dirichlet distribution for large mutation rate. Ann. Appl. Probab. 16 562-582. MR2244425 https://doi.org/10.1214/ 105051605000000818

Dawson, D. A. and Fleischmann, K. (1997). A continuous super-Brownian motion in a super-Brownian medium. J. Theoret. Probab. 10 213-276. MR1432624 https://doi.org/10.1023/A: 1022606801625

Dawson, D. A., Fleischmann, K. and Mörters, P. (2002). Strong clumping of super-Brownian motion in a stable catalytic medium. Ann. Probab. 30 1990-2045. MR1944014 https://doi.org/10.1214/aop/1039548380

DAWSON, D. A. and GÄRTNER, J. (1987). Large deviations from the McKean-Vlasov limit for weakly interacting diffusions. Stochastics 20 247-308. MR0885876 https://doi.org/10. 1080/17442508708833446

Dawson, D. A. and GorostizA, L. G. (1984). Limit theorems for supercritical branching random fields. Math. Nachr. 118 19-46. MR0773609 https://doi.org/10.1002/mana.19841180103

Dawson, D. A. and GorostizA, L. G. (2018). Transience and recurrence of random walks on percolation clusters in an ultrametric space. J. Theoret. Probab. 31 494-526. MR3769822 https://doi.org/10.1007/s10959-016-0691-7

Dawson, D. A., GorostizA, L. G. and Li, Z. (2002). Nonlocal branching superprocesses and some related models. Acta Appl. Math. 74 93-112. MR1936024 https://doi.org/10.1023/A: 1020507922973

Dawson, D. A., Gorostiza, L. G. and Wakolbinger, A. (2001). Occupation time fluctuations in branching systems. J. Theoret. Probab. 14 729-796. MR1860521 https://doi.org/10.1023/A: 1017597107544

Dawson, D. A., Gorostiza, L. G. and Wakolbinger, A. (2004). Hierarchical equilibria of branching populations. Electron. J. Probab. 9 316-381. MR2080603 https://doi.org/10.1214/EJP. v9-200

Dawson, D. A. and Greven, A. (1993). Hierarchical models of interacting diffusions: Multiple time scale phenomena, phase transition and pattern of cluster-formation. Probab. Theory Related Fields 96 435-473. MR1234619 https://doi.org/10.1007/ BF01200205

Dawson, D. A. and Greven, A. (1999). Hierarchically interacting Fleming-Viot processes with selection and mutation: Multiple space time scale analysis and quasi-equilibria. Electron. J. Probab. 4 no. 4, 81. MR1670873 https://doi.org/10.1214/EJP.v4-41

Dawson, D. A. and Greven, A. (2014). Spatial FlemingViot Models with Selection and Mutation. Lecture Notes in Math. 2092. Springer, Cham. MR3155790 https://doi.org/10.1007/ 978-3-319-02153-9

Dawson, D. A., Greven, A. and Vaillancourt, J. (1995). Equilibria and quasiequilibria for infinite collections of interacting Fleming-Viot processes. Trans. Amer. Math. Soc. 347 2277-2360. MR1297523 https://doi.org/10.2307/2154827

DAwson, D. A. and HochBERG, K. J. (1982). Wandering random measures in the Fleming-Viot model. Ann. Probab. 10 554-580. MR0659528
Dawson, D. A. and HochberG, K. J. (1983). Qualitative behavior of a selectively neutral allelic model. Theor. Popul. Biol. 23 1-18. https://doi.org/10.1016/0040-5809(83)90002-3

Dawson, D. A. and Kurtz, T. G. (1982). Applications of duality to measure-valued diffusion processes. In Advances in Filtering and Optimal Stochastic Control (Cocoyoc, 1982). Lect. Notes Control Inf. Sci. 42 91-105. Springer, Berlin. MR0794506 https://doi.org/10.1007/BFb0004528

DAwson, D. A. and LI, Z. (2012). Stochastic equations, flows and measure-valued processes. Ann. Probab. 40 813-857. MR2952093 https://doi.org/10.1214/10-AOP629

DAwson, D. A. and MARCH, P. (1995). Resolvent estimates for Fleming-Viot operators and uniqueness of solutions to related martingale problems. J. Funct. Anal. 132 417-472. MR1347357 https://doi.org/10.1006/jfan.1995.1111

Dawson, D. A. and Papanicolaou, G. C. (1984). A random wave process. Appl. Math. Optim. 12 97-114. MR0764811 https://doi.org/10.1007/BF01449037

DAwson, D. A. and Perkins, E. A. (1991). Historical processes. Mem. Amer. Math. Soc. 93 iv+179. MR1079034 https://doi.org/10. 1090/memo/0454

Dawson, D. A. and Perkins, E. A. (1998). Long-time behavior and coexistence in a mutually catalytic branching model. Ann. Probab. 26 1088-1138. MR1634416 https://doi.org/10.1214/aop/ 1022855746

DAWSON, D. A. and SALEHI, H. (1980). Spatially homogeneous random evolutions. J. Multivariate Anal. 10 141-180. MR0575923 https://doi.org/10.1016/0047-259X(80)90012-3

DAWsOn, D. A., TANG, J. and ZHAO, Y. Q. (2005). Balancing queues by mean field interaction. Queueing Syst. 49 335-361. MR2149648 https://doi.org/10.1007/s11134-005-6971-z

Dawson, D. A., Etheridge, A. M., Fleischmann, K., MytNIK, L., PER KINS, E. A. and XIONG, J. (2002). Mutually catalytic branching in the plane: Infinite measure states. Electron. J. Probab. 7 No. 15, 61. MR1921744 https://doi.org/10.1214/EJP.v7-114

Dawson, D. A., Greven, A., Den Hollander, F., Sun, R. and SWART, J. M. (2008). The renormalization transformation of twotype branching models. Ann. Inst. Henri Poincaré Probab. Stat. 44 1038-1077. MR2469334 https://doi.org/10.1214/07-AIHP143

Donnelly, P. and KURTZ, T. G. (1996). A countable representation of the Fleming-Viot measure-valued diffusion. Ann. Probab. 24 698-742. MR1404525 https://doi.org/10.1214/aop/1039639359

Duquesne, T. and Le GALl, J.-F. (2002). Random trees, Lévy processes and spatial branching processes. Astérisque 281 vi+147. MR1954248

DYNKIN, E. B. (1988). Representation for functionals of superprocesses by multiple stochastic integrals, with applications to selfintersection local times. Astérisque 157-158 147-171. MR0976217

DYNKIN, E. B. (1994). An Introduction to Branching Measure-Valued Processes. CRM Monograph Series 6. Amer. Math. Soc., Providence, RI. MR1280712 https://doi.org/10.1090/crmm/006

DYnkin, E. B. (2002). Diffusions, Superdiffusions and Partial Differential Equations. American Mathematical Society Colloquium Publications 50. Amer. Math. Soc., Providence, RI. MR1883198 https://doi.org/10.1090/coll/050

Etheridge, A. (2011). Some Mathematical Models from Population Genetics. Lecture Notes in Math. 2012. Springer, Heidelberg. MR2759587 https://doi.org/10.1007/978-3-642-16632-7

ETHERIDGE, A. and MARCH, P. (1991). A note on superprocesses. Probab. Theory Related Fields 89 141-147. MR1110534 https://doi.org/10.1007/BF01366902

Ethier, S. N. and Kurtz, T. G. (1986). Markov Processes: Characterization and Convergence. Wiley Series in Probability and Mathematical Statistics: Probability and Mathematical Statistics. Wiley, New York. MR0838085 https://doi.org/10.1002/9780470316658 
Evans, S. N. and Perkins, E. A. (1998). Collision local times, historical stochastic calculus, and competing superprocesses. Electron. J. Probab. 3 no. 5, 120. MR1615329 https://doi.org/10.1214/ EJP.v3-27

Genest, C. (1999). Probability and statistics: A tale of two worlds. Canad. J. Statist. 27 421-444. https://doi.org/10.2307/3315650

Genest, C. and GuAY, M. (2002). Worldwide research output in probability and statistics: An update. Canad. J. Statist. 30 329-342. MR1930515 https://doi.org/10.2307/3315955

ISCOE, I. (1981). The man-hour process associated with measure valued branching random motions in $R^{D}$. Ph.D. thesis. MR2631942

ISCOE, I. (1986a). Ergodic theory and a local occupation time for measure-valued critical branching Brownian motion. Stochastics 18 197-243. MR0861108 https://doi.org/10.1080/ 17442508608833409

IsCOE, I. (1986b). A weighted occupation time for a class of measurevalued branching processes. Probab. Theory Related Fields 71 85116. MR0814663 https://doi.org/10.1007/BF00366274

ISCOE, I. (1988). On the supports of measure-valued critical branching Brownian motion. Ann. Probab. 16 200-221. MR0920265

Itô, K. and McKean, H. P. JR. (1974). Diffusion Processes and Their Sample Paths. Die Grundlehren der Mathematischen Wissenschaften 125. Springer, Berlin. MR0345224

JAGERS, P. (1974). Aspects of random measures and point processes. In Advances in Probability and Related Topics, Vol. 3 179-239. MR0397872

KALlEnBERG, O. (1978). On conditional intensities of point processes. Z. Wahrsch. Verw. Gebiete 41 205-220. MR0461654 https://doi.org/10.1007/BF00534240

Kingman, J. F. C. (1976). Coherent random walks arising in some genetical models. Proc. R. Soc. Lond., Ser. A 351 19-31. MR0420867 https://doi.org/10.1098/rspa.1976.0127

KonnO, N. and SHIGA, T. (1988). Stochastic partial differential equations for some measure-valued diffusions. Probab. Theory
Related Fields 79 201-225. MR0958288 https://doi.org/10.1007/ BF00320919

LE GALL, J.-F. (1991). Brownian excursions, trees and measurevalued branching processes. Ann. Probab. 19 1399-1439. MR1127710

Matthes, K., Kerstan, J. and Mecke, J. (1978). Infinitely Divisible Point Processes. Wiley Series in Probability and Mathematical Statistics. Wiley, Chichester-New York-Brisbane. MR0517931

PERKINS, E. A. (1988). A space-time property of a class of measurevalued branching diffusions. Trans. Amer. Math. Soc. 305 743-795. MR0924777 https://doi.org/10.2307/2000886

PERKINS, E. (1989). The Hausdorff measure of the closed support of super-Brownian motion. Ann. Inst. Henri Poincaré Probab. Stat. 25 205-224. MR1001027

Perkins, E. (1990). Polar sets and multiple points for superBrownian motion. Ann. Probab. 18 453-491. MR1055416

PERKINS, E. (2002). Dawson-Watanabe superprocesses and measurevalued diffusions. In Lectures on Probability Theory and Statistics (Saint-Flour, 1999). Lecture Notes in Math. 1781 125-324. Springer, Berlin. MR1915445

ShigA, T. and ShimizU, A. (1980). Infinite-dimensional stochastic differential equations and their applications. J. Math. Kyoto Univ. 20 395-416. MR0591802 https://doi.org/10.1215/kjm/1250522207

Shiga, T. and UchiYAma, K. (1986). Stationary states and their stability of the stepping stone model involving mutation and selection. Probab. Theory Related Fields 73 87-117. MR0849066 https://doi.org/10.1007/BF01845994

WALSH, J. B. (1986). An introduction to stochastic partial differential equations. In École D'été de Probabilités de Saint-Flour, XIV1984. Lecture Notes in Math. 1180 265-439. Springer, Berlin. MR0876085 https://doi.org/10.1007/BFb0074920

WATANABE, S. (1968). A limit theorem of branching processes and continuous state branching processes. J. Math. Kyoto Univ. $8141-$ 167. MR0237008 https://doi.org/10.1215/kjm/1250524180 